\title{
A New Generalization of Quadratic Hazard Rate Distribution
}

Ibrahim Elbatal

Institute of Statistical Studies and Research

Department of Mathematical

Statistics, Cairo University

i_elbatal@staff.cu.edu.eg

Nadeem Shafique Butt

COMSATS Institute of Information Technology, Lahore

nadeemshafique@ ciitlahore.edu.pk

\begin{abstract}
For the first time, a five-parameter distribution, called the kumaraswamy quadratic hazard rate distribution is defined and studied. The new distribution contains as special models some well-known distributions discussed in lifetime literature, such as the Linear failure rate, Exponential and Rayleigh distributions, among several others. We obtain the moments, moment generating and quantile functions. We discuss the method of maximum likelihood to estimate the model parameters and determine the observed information matrix. A real data sets illustrate the importance and flexibility of the proposed models.
\end{abstract}

Keywords: Quadratic Hazard Rate distribution, Order Statistics,Maximum Likelihood Estimation, Reliability Function.

\section{Introduction}

The quadratic hazard rate distribution $(Q H R)$ distribution was introduced by Bain (1974). This distribution generalizes several well known distributions. Among these distributions are the linear fialure (hazard) rate, exponential and Rayleigh distributions. Also, the $Q H R D$ may have an increasing (decreasing) hazard function or a bathtub shaped hazard function or an upsidedown bathtub shaped hazard function. This property enables this distribution to be used in many applications in several areas, such as reliability, life testing, survival analysis and others.

A random variable $X$ is said to have the quadratic hazard rate distribution $(Q H R D)$ with three parameters $\alpha, \theta$, and $\beta$, if it has the cumulative distribution function

$$
G(x, \alpha, \theta, \beta)=1-e^{-\left(\alpha x+\frac{\theta}{2} x^{2}+\frac{\beta}{3} x^{3}\right)}, x>0,
$$

where $\alpha \geq 0, \beta \geq 0$ and $\theta \geq-2 \sqrt{\alpha \beta}$. This restriction on the parameter space is made to be insure that the hazard function with the following form is positive, see Bain (1974), $A(x, \alpha, \theta, \beta)=\alpha+\theta x+\beta x^{2}, x>0$. The corresponding probability density function (pdf) is given by

$$
g(x, \alpha, \theta, \beta)=\left(\alpha+\theta x+\beta x^{2}\right) e^{-\left(\alpha x+\frac{\theta}{2} x^{2}+\frac{\beta}{3} x^{3}\right)}, x>0,
$$


Distribution generalization theory has been the focus of prominent investigation over the past years (see, e.g., Amoroso 1925; Good 1953; Hoskings and Wallis 1987; McDonald 1984; Gupta et al. 1998). A particular generalization model is the class of beta generalized distributions introduced in Eugene et al. (2002) generated from the logit of the beta random variable. Recently, Cordeiro and Castro (2011) introduced a new family of generalized distributions (Kw-G), based on the Kumaraswamy distribution (Kumaraswamy 1980), to extend the $K_{w}$ - normal distribution is obtained by taking $G(x)$ in (1.2) to be the normal cumulative function. Analogously, the $K_{w}$-Weibull (Cordeiro et al. (2010)), General results for the Kumaraswamy- $G$ distribution (Nadarajah et al. (2012)). $K_{w}$ - generalized gamma (Pascoa et al. (2011)), Kw- Birnbaum-Saunders (Saulo et al. (2012)), and $K_{w}-$ Gumbel (Cordeiro et al. (2012)) distributions are obtained by taking $G(x)$ to be the cdf of the Weibull, generalized gamma, Birnbaum-Saunders and Gumbel distributions, respectively, Elbatal (2013) introduced kumaraswamy generalized linear failure rate , and the Kumaraswamy Exponentiated Pareto Distribution, among several others. Hence, each new $K w-G$ distribution can be generated from a specified $G$ distribution.

The Kumaraswamy $\left(K_{w}\right)$ distribution is not very common among statisticians and has been little explored in the literature.The cdf and pdf of the Kumaraswamy distribution are defined by

$$
F_{X \mid(a, b)}(x)=1-\left(1-x^{a}\right)^{b}, 0<x<1,
$$

where $a>0$ and $b>0$ are shape parameters, and the probability density function

$$
f_{X \mid(a, b)}(x)=a b x^{a-1}\left(1-x^{a}\right)^{b-1}
$$

which can be unimodal, increasing, decreasing or constant, depending on the parameter values. It does not seem to be very familiar to statisticians and has not been investigated systematically in much detail before, nor has its relative interchangeability with the beta distribution been widely appreciated. However, in a very recent paper, Jones (2009) explored the background and genesis of this distribution and, more importantly, made clear some similarities and differences between the beta and $K_{w}$ distributions. However, the beta distribution has the following advantages over the $K_{w}$ distribution: simpler formulae for moments and moment generating function (mgf), a one-parameter sub-family of symmetric distributions, simpler moment estimation and more ways of generating the distribution by means of physical processes.

In this note, we combine the works of Kumaraswamy (1980) and Cordeiro and Castro (2011) to derive some mathematical properties of a new model, called the Kumaraswamy quadratic hazard rate $(K Q H R)$ distribution, which stems from the following general construction: if $G$ denotes the baseline cumulative function of a random variable, then a generalized class of distributions can be defined by

$$
F_{X \mid(a, b)}(x)=1-\left[1-G(x)^{a}\right]^{b}
$$


where $a>0$ and $b>0$ are two additional shape parameters. The $K w-G$ distribution can be used quite effectively even if the data are censored. Correspondingly, its density function is distributions has a very simple form

$$
f_{X \mid(a, b)}(x)=\operatorname{abg}(x) G(x)^{a-1}\left[1-G(x)^{a}\right]^{b-1}
$$

The density family (1.6) has many of the same properties of the class of beta- $G$ distributions (see Eugene et al. (2002)), but has some advantages in terms of tractability, since it does not involve any special function such as the beta function. A physical interpretation of the $K_{w}-G$ distribution given by (1.5) and (1.6) (for $a$ and $b$ positive integers) is as follows. Suppose a system is made of $b$ independent components and that each component is made up of $a$ independent subcomponents. Suppose the system fails if any of the $b$ components fails and that each component fails if all of the $a$ subcomponents fail. Let $X_{j 1}, X_{j 2}, \ldots, X_{j a}$ denote the life times of the subcomponents with in the $j_{t h}$ component, $j=1, \ldots, b$ with common (cdf) $G$. Let $X_{j}$ denote the lifetime of the $j_{t h}$ component, $j=1, \ldots, b$ and let $X$ denote the lifetime of the entire system. Then the (cdf) of $X$ is given by

$$
\begin{aligned}
& P(X \leq x)=1-P\left(X_{1}>x, X_{2}>x, \ldots, X_{b}>x\right) \\
& \quad=1-\left[P\left(X_{1}>x\right)\right]^{b}=1-\left\{1-P\left(X_{1} \leq x\right\}^{b}\right. \\
& \quad=1-\left\{1-P\left(X_{11} \leq x, X_{12} \leq x, \ldots, X_{1 a} \leq x\right\}^{b}\right. \\
& \quad=1-\left\{1-P\left[X_{11} \leq x\right]^{a}\right\}^{b}=1-\left\{1-G^{a}(x)\right\}^{b} .
\end{aligned}
$$

So, it follows that the $K_{w}-G$ distribution given by (1.5) and (1.6) is precisely the time to failure distribution of the entire system.

The rest of the article is organized as follows. In Section 2, we define the cumulative, density and hazard functions of the KQHR distribution and some special cases. In Section 3. includes $r_{t h}$ moment, moment generating function. The distribution of the order statistics are proposed in Section4. Least squares and weighted least squares estimators introduced in Section 5. Finally, maximum likelihood estimation is performed in Section 6.

\section{Kumaraswamy Quadratic Hazard Rate Distribution}


In this section we studied the kumaraswamy quadratic hazard rate $(K Q H R)$ distribution and the sub-models of this distribution. Now using (1.1) and (1.2) in (1.5) we have the cdf of Kumaraswamy quadratic hazard rate distribution

$$
F_{K Q H R}(x)=1-\left\{1-\left[1-e^{-\left(\alpha x+\frac{\theta}{2} x^{2}+\frac{\beta}{3} x^{3}\right)}\right]^{a}\right\}^{b} \text {. }
$$

The corresponding probability density function (pdf) of the kumaraswamy quadratic hazard rate distribution is given by

$$
f_{\text {KQHR }}(x)=a b\left(\alpha+\theta x+\beta x^{2}\right) e^{-\left(\alpha x+\frac{\theta}{2} x^{2}+\frac{\beta}{3} x^{3}\right)}\left[1-e^{-\left(\alpha x+\frac{\theta}{2} x^{2}+\frac{\beta}{3} x^{3}\right)}\right]^{a-1}\left\{1-\left[1-e^{-\left(\alpha x+\frac{\theta}{2} x^{2}+\frac{\beta}{3} x^{3}\right)}\right]^{a}\right\}^{b-1} .
$$

Graph of (2.2) for various values of $a, b, \alpha, \beta$ and $\theta$ is given in appendix-I

The Linear failure rate (LFR), exponential (E), Rayleigh (R) distributions are clearly the most important submodels of Equation (2.2). Other submodels can be immeditely defined from Table 1. It is evident that (2.2) is much more flexible than the different distributions listed in Table1. The following are special cases of the $\operatorname{KQHR}(\alpha, \theta, \beta, a, b)$ :

Table 1: Some sub-models of the $K Q H R$ distribution

\begin{tabular}{|l|c|c|c|c|c|}
\hline Distribution & $\boldsymbol{\alpha}$ & $\boldsymbol{\theta}$ & $\boldsymbol{\beta}$ & $\boldsymbol{A}$ & $\boldsymbol{b}$ \\
\hline Quadratic Hazard Rate & - & - & - & 1 & 1 \\
\hline kumaraswamy Linear failure rate & - & - & 0 & - & - \\
\hline kumaraswamy Rayleigh & 0 & - & 0 & - & - \\
\hline kumaraswamy Exponential & - & 0 & 0 & - & - \\
\hline Linear failure rate & - & - & 0 & 1 & 1 \\
\hline Rayleigh & 0 & - & 0 & 1 & 1 \\
\hline Exponential & - & 0 & 0 & 1 & 1 \\
\hline
\end{tabular}

\subsection{Reliability Analysis}

The Kumaraswamy quadratic hazard rate can be a useful characterization of life time data analysis. The reliability function $(R F)$ of the Kumaraswamy quadratic hazard rate distribution is denoted by $R_{K Q H R}(x)$ also known as the survivor function and is defined as

$$
R_{K Q H R}(x)=1-F_{K Q H R}(x)=\left\{1-\left[1-e^{-\left(\alpha x+\frac{\theta}{2} x^{2}+\frac{\beta}{3} x^{3}\right)}\right]^{a}\right\}^{b} .
$$


It is important to note that $R_{K Q H R}(x)+F_{K Q H R}(x)=1$. The hazard rate function (HF) and reversed hazarde rate function defined by

$$
\begin{aligned}
& h_{K Q H R}(x)=\frac{f_{K Q H R}(x)}{1-F_{K Q H R}(x)} \\
& =\frac{a b\left(\alpha+\theta x+\beta x^{2}\right) e^{-\left(\alpha x+\frac{\theta}{2} x^{2}+\frac{\beta}{3} x^{3}\right)\left[1-e^{\left.-\left(\alpha x+\frac{\theta}{2} x^{2}+\frac{\beta}{3} x^{3}\right)\right]^{a-1}}\right]}}{\left\{1-\left[1-e^{\left.-\left(\alpha x+\frac{\theta}{2} x^{2}+\frac{\beta}{3} x^{3}\right)\right]^{a}}\right\} .\right.}
\end{aligned}
$$

Graph of (2.4) for various values of $a, b, \alpha, \beta$ and $\theta$ is given in appendix-II

and

$$
\begin{aligned}
& \tau_{K Q H R}(x)=\frac{f_{K Q H R}(x)}{F_{K Q H R}(x)} \\
& =\frac{a b\left(\alpha+\theta x+\beta x^{2}\right) e^{-\left(\alpha x+\frac{\theta}{2} x^{2}+\frac{\beta}{3} x^{3}\right)}}{1-\left\{1-\left[1-e^{-\left(\alpha x+\frac{\theta}{2} x^{2}+\frac{\beta}{3} x^{3}\right)}\right]^{a}\right\}^{b}} \times\left[1-e^{-\left(\alpha x+\frac{\theta}{2} x^{2}+\frac{\beta}{3} x^{3}\right)}\right]^{a-1}\left\{1-\left[1-e^{-\left(\alpha x+\frac{\theta}{2} x^{2}+\frac{\beta}{3} x^{3}\right)}\right]\right]^{a},
\end{aligned}
$$

respectively. It is important to note that the units for $h_{K Q H R}(x)$ is the probability of failure per unit of time, distance or cycles. These failure rates are defined with different choices of parameters. The cumulative hazard function of the Kumaraswamy quadratic hazard rate distribution is denoted by $H_{K Q H R}(x)$ and is defined as

$$
H_{K Q H R}(x)=-\ln \left|1-\left\{1-\left[1-e^{-\left(\alpha x+\frac{\theta}{2} x^{2}+\frac{\beta}{3} x^{3}\right)}\right]^{a}\right\}\right|
$$

It is important to note that the units for $H_{K Q H R}(x)$ is the cumulative probability of failure per unit of time, distance or cycles. we can show that . For all choice of parameters the distribution has the decreasing patterns of cumulative instantaneous failure rates. 


\section{Statistical Properties}

In this section we study the statistical properties of the kumaraswamy quadratic hazard rate distribution. Specifically quantile, moments and moment generating function. Moments are necessary and important in any statistical analysis, especially in applications. It can be used to study the most important features and characteristics of a distribution (e.g., tendency, dispersion, skewness and kurtosis).

\section{Quantile and Random Number Generation}

The quantile $x_{q}$ of the $K Q H R(\alpha, \theta, \beta, a, b)$ is real solution of the following equation

$$
{ }_{\alpha x_{q}}+\frac{\theta}{2} x_{q}^{2}+\frac{\beta}{3} x_{q}^{3}+\ln \left[1-(1-q)^{\frac{1}{b}}\right]^{\frac{1}{a}}=0
$$

The random number generation as $x$ of the $\operatorname{KQHR}(\alpha, \theta, \beta, a, b)$ is defined by the following relation

$$
{ }_{\alpha x_{q}}+\frac{\theta}{2} x_{q}^{2}+\frac{\beta}{3} x_{q}^{3}+\ln \left[1-(1-\varphi)^{\frac{1}{b}}\right]^{\frac{1}{a}}=0, \text { where } \varphi \sim U(0,1)
$$

\section{Moments}

The following theorems give the $r_{t h}$ moment $\left(\mu_{r}\right)$ and moment generating function $M_{X}(t)$ of the $\operatorname{KQHR}(x ; \alpha, \theta, \beta, a, b)$.

\section{Theorem (3.1)}

If $X$ has the $K Q H R(x ; \alpha, \theta, \beta, a, b)$, then the $r_{t h}$ moment of $X$ is given by the following

$$
\begin{aligned}
\mu_{r}= & \sum_{i, j, k, m=0}^{\infty}(-1)^{i+j+k+m}\left(\begin{array}{c}
b-1 \\
i
\end{array}\right)\left(\begin{array}{c}
a(i+1)-1 \\
j
\end{array}\right) \frac{\theta^{k} \beta^{m}(j+1)^{k}(j+1)^{m}}{2^{k} 3^{m} k ! m !} \\
& \times\left[\frac{\alpha \Gamma(r+2 k+3 m+1)}{[\alpha(j+1)]^{r+2 k+3 m+1}}+\theta \frac{\Gamma(r+2 k+3 m+2)}{[\alpha(j+1)]^{r+2 k+3 m+2}}+\frac{\beta \Gamma(r+2 k+3 m+3)}{[\alpha(j+1)]^{r+2 k+3 m+3}}\right]
\end{aligned}
$$

Proof:

Starting with

$$
\begin{aligned}
& \mu_{r}^{\prime}=\int_{0}^{\infty} x^{r} f_{K Q H R}(x) d x=\int_{0}^{\infty} x^{r} a b\left(\alpha+\theta x+\beta x^{2}\right) e^{-\left(\alpha x+\frac{\theta}{2} x^{2}+\frac{\beta}{3} x^{3}\right)} \\
& \times\left[1-e^{-\left(\alpha x+\frac{\theta}{2} x^{2}+\frac{\beta}{3} x^{3}\right)}\right]^{a-1}\left\{1-\left[1-e^{-\left(\alpha x+\frac{\theta}{2} x^{2}+\frac{\beta}{3} x^{3}\right)}\right]^{a}\right\}^{b-1} d x .
\end{aligned}
$$


since $0<e^{-\left(\alpha x+\frac{\theta}{2} x^{2}+\frac{\beta}{3} x^{3}\right)}<1$, then the using the binomial of $\left\{1-\left[1-e^{-\left(\alpha x+\frac{\theta}{2} x^{2}+\frac{\beta}{3} x^{3}\right)}\right]^{a}\right\}^{b-1}$ given

by

$$
\left\{1-\left[1-e^{-\left(\alpha x+\frac{\theta}{2} x^{2}+\frac{\beta}{3} x^{3}\right)}\right]^{a}\right\}^{b-1}=\sum_{i=0}^{\infty}(-1)^{i}\left(\begin{array}{c}
b-1 \\
i
\end{array}\right)\left[1-e^{-\left(\alpha x+\frac{\theta}{2} x^{2}+\frac{\beta}{3} x^{3}\right)}\right]^{a i},
$$

then

$$
\mu_{r}=\sum_{i=0}^{\infty}(-1)^{i}\left(\begin{array}{c}
b-1 \\
i
\end{array}\right) a b \int_{0}^{\infty} x^{r}\left(\alpha+\theta x+\beta x^{2}\right) e^{-\left(\alpha x+\frac{\theta}{2} x^{2}+\frac{\beta}{3} x^{3}\right)}\left[1-e^{-\left(\alpha x+\frac{\theta}{2} x^{2}+\frac{\beta}{3} x^{3}\right)}\right]^{a(i+1)-1} d x
$$

again

$$
\left[1-e^{-\left(\alpha x+\frac{\theta}{2} x^{2}+\frac{\beta}{3} x^{3}\right)}\right]^{a(i+1)-1}=\sum_{j=0}^{\infty}(-1)^{j}\left(\begin{array}{c}
a(i+1)-1 \\
j
\end{array}\right) e^{-\left(\alpha x+\frac{\theta}{2} x^{2}+\frac{\beta}{3} x^{3}\right) j}
$$

therefore

$$
\begin{aligned}
\mu_{r}^{\prime}= & \sum_{i=0}^{\infty} \sum_{j=0}^{\infty}(-1)^{i+j}\left(\begin{array}{c}
b-1 \\
i
\end{array}\right)\left(\begin{array}{c}
a(i+1)-1 \\
j
\end{array}\right) a b \\
& \times \int_{0}^{\infty} x^{r}\left(\alpha+\theta x+\beta x^{2}\right) e^{-(j+1)\left(\alpha x+\frac{\theta}{2} x^{2}+\frac{\beta}{3} x^{3}\right)} d x,
\end{aligned}
$$

but the expansion of $e^{-(i+1) \frac{\theta}{2} x^{2}}$ and $e^{-(i+1) \frac{\beta}{3} x^{3}}$ are given by

$$
e^{-(j+1) \frac{\theta}{2} x^{2}}=\sum_{k=0}^{\infty} \frac{\left(-(j+1) \frac{\theta}{2} x^{2}\right)^{k}}{k !} \text { and } e^{-(j+1) \frac{\beta}{3} x^{3}}=\sum_{m=0}^{\infty} \frac{\left(-(j+1) \frac{\beta}{3} x^{3}\right)^{m}}{m !},
$$

substituting from (3.7) into (3.6) we have

$$
\begin{aligned}
\mu_{r}^{\prime} & =W_{i, j, k, m}\left\{\alpha \int_{0}^{\infty} x^{r+2 k+3 m} e^{-\alpha(j+1) x} d x+\theta \int_{0}^{\infty} x^{r+2 k+3 m+1} e^{-\alpha(j+1) x} d x\right. \\
& \left.+\beta \int_{0}^{\infty} x^{r+2 k+3 m+2} e^{-\alpha(j+1) x} d x\right\} \\
= & W_{i, j, k, m}\left[\frac{\alpha \Gamma(r+2 k+3 m+1)}{[\alpha(j+1)]^{r+2 k+3 m+1}}+\theta \frac{\Gamma(r+2 k+3 m+2)}{[\alpha(j+1)]^{r+2 k+3 m+2}}+\frac{\beta \Gamma(r+2 k+3 m+3)}{[\alpha(j+1)]^{r+2 k+3 m+3}}\right],
\end{aligned}
$$


where

$$
W_{i, j, k, m}=\sum_{i=j=k=m=0}^{\infty}(-1)^{i+j+k+m}\left(\begin{array}{c}
b-1 \\
i
\end{array}\right)\left(\begin{array}{c}
a(i+1)-1 \\
j
\end{array}\right) \frac{\theta^{k} \beta^{m}(j+1)^{k}(j+1)^{m}}{2^{k} 3^{m} k ! m !} .
$$

Which completes the proof.

Based on Theorem (3.1) the measures of variation, skewness and kurtosis of the $K Q H R$ $(\alpha, \theta, \beta, \mu, a, b)$ distribution can be obtained according to the following relation

$$
\begin{aligned}
& C V_{K Q H R}=\sqrt{\frac{\mu_{2}}{\mu_{1}}-1}, \\
& C S_{K Q H R}=\frac{\mu_{3}(\theta)-3 \mu_{1}(\theta) \mu_{2}(\theta)+2 \mu_{1}^{3}(\theta)}{\left[\mu_{2}(\theta)-\mu_{1}^{2}(\theta)\right]^{\frac{3}{2}}} \\
& \text { and } C K_{K Q H R}=\frac{\mu_{4}(\theta)-4 \mu_{1}(\theta) \mu_{3}(\theta)+6 \mu_{1}^{2}(\theta) \mu_{2}(\theta)-3 \mu_{1}^{4}(\theta)}{\left[\mu_{2}(\theta)-\mu_{1}^{2}(\theta)\right]^{2}} .
\end{aligned}
$$

\subsection{Moment Generating Function}

In this subsection we derived the moment generating function (mgf) of kumaraswamy quadratic hazard rate distribution.

Theorem (3.2): If $X$ has the $\operatorname{KQHR}(\alpha, \theta, \beta, \mu, a, b)$, then the the moment generating function (mgf) of $X$ is given as follows

$$
M_{X}(t)=W_{i, j, k, m}\left[\frac{\alpha \Gamma(2 k+3 m+1)}{[\alpha(j+1)-t]^{2 k+3 m+1}}+\theta \frac{\Gamma(2 k+3 m+2)}{[\alpha(j+1)-t]^{2 k+3 m+2}}+\frac{\beta \Gamma(2 k+3 m+3)}{[\alpha(j+1)-t]^{2 k+3 m+3}}\right]
$$

Proof:

$$
\begin{aligned}
M_{X}(t) & =\int_{0}^{\infty} e^{t x} f_{K Q H R}(x) d x=\int_{0}^{\infty} e^{t x} a b\left(\alpha+\theta x+\beta x^{2}\right) e^{-\left(\alpha x+\frac{\theta}{2} x^{2}+\frac{\beta}{3} x^{3}\right)} \\
\times & {\left[1-e^{-\left(\alpha x+\frac{\theta}{2} x^{2}+\frac{\beta}{3} x^{3}\right)}\right]^{a-1}\left\{1-\left[1-e^{-\left(\alpha x+\frac{\theta}{2} x^{2}+\frac{\beta}{3} x^{3}\right)}\right]^{a}\right\}^{b-1} d x }
\end{aligned}
$$

substituting (3.3), (3.4)and (3.5) into relation (3.9) we get the following

$$
M_{X}(t)=W_{i, j, k, m} \int_{0}^{\infty} x^{2 k+3 m}\left(\alpha+\theta x+\beta x^{2}\right) e^{-(\alpha(i+1)-t) x} d x
$$




$$
\begin{aligned}
& =W_{i, j, k, m}\left\{\alpha \int_{0}^{\infty} x^{2 k+3 m} e^{-(\alpha(j+1)-t) x} d x+\theta \int_{0}^{\infty} x^{2 k+3 m+1} e^{-(\alpha(j+1)-t) x} d x+\beta \int_{0}^{\infty} x^{2 k+3 m+2} e^{-(\alpha(j+1)-t) x} d x\right\} \\
& =W_{i, j, k, m}\left[\frac{\alpha \Gamma(2 k+3 m+1)}{[\alpha(j+1)-t]^{2 k+3 m+1}}+\theta \frac{\Gamma(2 k+3 m+2)}{[\alpha(j+1)-t]^{2 k+3 m+2}}+\frac{\beta \Gamma(2 k+3 m+3)}{[\alpha(j+1)-t]^{2 k+3 m+3}}\right] .
\end{aligned}
$$

Which completes the proof.

\section{Order Statistics}

Moments of order statistics play an important role in quality control testing and reliability, where a practitioner needs to predict the failure of future items based on the times of a few early failures. These predictors are often based on moments of order statistics. We now derive an explicit expression for the density function of the $i_{t h}$ order statistic $X_{(i: n)}$, say $f_{i: n}(x)$, in a random sample of size $n$ from the $K Q H R$ distribution. Let $X_{1}, X_{2}, \ldots, X_{n}$ be a simple random sample from $K Q H R(x ; \alpha, \theta, \beta, a, b)$ with cumulative distribution function and probability density function as in (2.1) and (2.2), respectively. Let $X_{(1: n)} \leq X_{(2: n)} \leq \ldots \leq X_{(n: n)}$ denote the order statistics obtained from this sample. In reliability literature, $X_{(i: n)}$ denote the lifetime of an $(n-i+1)-$ out - of $-n$ system which consists of $n$ independent and identically components. Then the pdf of $X_{(i: n)}, 1 \leq i \leq n$ is given by

$$
f_{i: n}(x)=\frac{1}{\beta(i, n-i+1)}[F(x, \Phi)]^{i-1}[1-F(x, \Phi)]^{n-i} f(x, \Phi)
$$

where $\Phi=(\alpha, \theta, \beta, a, b)$. We defined the first order statistics $X_{(1)}=\operatorname{Min}\left(X_{1}, X_{2}, \ldots, X_{n}\right)$, the the last order statistics as $X_{(n)}=\operatorname{Max}\left(X_{1}, X_{2}, \ldots, X_{n}\right)$ and median order $X_{m+1}$.

The pdf of the $i_{t h}$ order statistic for Kumaraswamy quadratic hazard rate distribution is given by

$$
\begin{gathered}
f_{i: n}(x)=\frac{a b}{\beta(i, n-i+1)}\left[1-\left\{1-\left[1-e^{-\left(\alpha x+\frac{\theta}{2} x^{2}+\frac{\beta}{3} x^{3}\right)}\right]^{a}\right\}^{b}\right]^{i-1}\left[\left\{1-\left[1-e^{-\left(\alpha x+\frac{\theta}{2} x^{2}+\frac{\beta}{3} x^{3}\right)}\right]^{a}\right\}^{b}\right]^{n-i} \\
\times\left(\alpha+\theta x+\beta x^{2}\right) e^{-\left(\alpha x+\frac{\theta}{2} x^{2}+\frac{\beta}{3} x^{3}\right)\left[1-e^{-\left(\alpha x+\frac{\theta}{2} x^{2}+\frac{\beta}{3} x^{3}\right)}\right]^{a-1}\left\{1-\left[1-e^{-\left(\alpha x+\frac{\theta}{2} x^{2}+\frac{\beta}{3} x^{3}\right)}\right]^{a}\right\}^{b-1},}
\end{gathered}
$$

Therefore, the pdf of the largest order statistic $X_{(n)}$ is given by 


$$
\begin{aligned}
& f_{n: n}(x)=a b n\left[1-\left\{1-\left[1-e^{-\left(\alpha x+\frac{\theta}{2} x^{2}+\frac{\beta}{3} x^{3}\right)}\right]^{a}\right\}^{b}\right]^{n-1}\left(\alpha+\theta x+\beta x^{2}\right) e^{-\left(\alpha x+\frac{\theta}{2} x^{2}+\frac{\beta}{3} x^{3}\right)} \\
& \times\left[1-e^{-\left(\alpha x+\frac{\theta}{2} x^{2}+\frac{\beta}{3} x^{3}\right)}\right]^{a-1}\left\{1-\left[1-e^{-\left(\alpha x+\frac{\theta}{2} x^{2}+\frac{\beta}{3} x^{3}\right)}\right]^{a}\right\}^{b-1},
\end{aligned}
$$

and the pdf of the smallest order statistic $X_{(1)}$ is given by

$$
\begin{gathered}
f_{1: n}(x)=\operatorname{abn}\left[\left\{1-\left[1-e^{-\left(\alpha x+\frac{\theta}{2} x^{2}+\frac{\beta}{3} x^{3}\right)}\right]^{a}\right]^{b}\right]^{n-1} \\
\times\left[1-e^{-\left(\alpha x+\frac{\theta}{2} x^{2}+\frac{\beta}{3} x^{3}\right)}\right]^{a-1}\left\{1-\left[1-e^{-\left(\alpha x+\frac{\theta}{2} x^{2}+\frac{\beta}{3} x^{3}\right)}\right]^{a}\right\}^{b-1} .
\end{gathered}
$$

\section{Least Squares and Weighted Least Squares Estimators}

In this section we provide the regression based method estimators of the unknown parameters of the kumaraswamy quadratic hazard rate distribution, which was originally suggested by Swain, Venkatraman and Wilson (1988) to estimate the parameters of beta distributions. It can be used some other cases also. Suppose $Y_{1}, \ldots, Y_{n}$ is a random sample of size $n$ from a distribution function $G($.$) and suppose Y_{(i)} ; i=1,2, \ldots, n$ denotes the ordered sample. The proposed method uses the distribution of $G\left(Y_{(i)}\right)$. For a sample of size $n$, we have

$$
\begin{aligned}
& E\left(G\left(Y_{(i)}\right)\right)=\frac{i}{n+1}, V\left(G\left(Y_{(i)}\right)\right)=\frac{i(n-i+1)}{(n+1)^{2}(n+2)} \\
& \text { and } \operatorname{Cov}\left(G\left(Y_{(i)}\right), G\left(Y_{(j)}\right)\right)=\frac{i(n-j+1)}{(n+1)^{2}(n+2)} ; \text { for } i<j,
\end{aligned}
$$

see Johnson, Kotz and Balakrishnan (1995). Using the expectations and the variances, two variants of the least squares methods can be used.

Method 1 (Least Squares Estimators). Obtain the estimators by minimizing 


$$
Q(\alpha, \theta, \beta, a, b)=\sum_{i=1}^{n}\left(G\left(Y_{(i)}\right)-\frac{i}{n+1}\right)^{2},
$$

with respect to the unknown parameters. Therefore in case of $K Q H R$ distribution the least squares estimators of $\alpha, \theta, \beta, a$ and $b$, say $\alpha_{L S E}, \theta_{L S E}, \beta_{L S E}, a_{L S E}$ and $\hat{b}_{L S E}$ respectively, by using (2.1) and (5.1) we have the following equation

$$
Q(\alpha, \theta, \beta, a, b)=\sum_{i=1}^{n}\left[1-\left\{1-\left[1-e^{-\left(\alpha x_{(i)}+\frac{\theta}{2} x_{(i)}^{2}+\frac{\beta}{3} x_{(i)}^{3}\right)}\right]^{a}\right\}^{b}-\frac{i}{n+1}\right]^{2}
$$

To minimize equation (5.2) with respect to $\alpha, \theta, \beta, a$ and $b$ and, we differentiate with respect to these parameters, which leads to the following equations.

$$
\begin{aligned}
& \frac{\partial Q}{\partial \alpha}=a b x_{(i)}\left[1-\left\{1-\left[1-e^{-\left(\alpha x_{(i)}+\frac{\theta}{2} x_{(i)}^{2}+\frac{\beta}{3} x_{(i)}^{3}\right)}\right]^{a}\right\}^{b}-\frac{i}{n+1}\right] e^{-\left(\alpha x_{(i)}+\frac{\theta}{2} x_{(i)}^{2}+\frac{\beta}{3} x_{(i)}^{3}\right)} \\
& \times\left\{1-\left[1-e^{-\left(\alpha x_{(i)}+\frac{\theta}{2} x_{(i)}^{2}+\frac{\beta}{3} x_{(i)}^{3}\right)}\right]^{a}\right\}^{b-1}\left[1-e^{-\left(\alpha x_{(i)}+\frac{\theta}{2} x_{(i)}^{2}+\frac{\beta}{3} x_{(i)}^{3}\right)}\right]^{a-1}, \\
& \frac{\partial Q}{\partial \theta}=\frac{a b x_{(i)}^{2}}{2}\left[1-\left\{1-\left[1-e^{-\left(\alpha x_{(i)}+\frac{\theta}{2} x_{(i)}^{2}+\frac{\beta}{3} x_{(i)}^{3}\right)}\right]^{a}\right\}^{b}-\frac{i}{n+1}\right] e^{-\left(\alpha x_{(i)}+\frac{\theta}{2} x_{(i)}^{2}+\frac{\beta}{3} x_{(i)}^{3}\right)} \\
& \times\left\{1-\left[1-e^{-\left(\alpha x_{(i)}+\frac{\theta}{2} x_{(i)}^{2}+\frac{\beta}{3} x_{(i)}^{3}\right)}\right]^{a}\right\}^{b-1}\left[1-e^{-\left(\alpha x_{(i)}+\frac{\theta}{2} x_{(i)}^{2}+\frac{\beta}{3} x_{(i)}^{3}\right)}\right]^{a-1}
\end{aligned}
$$




$$
\begin{aligned}
& \frac{\partial Q}{\partial \beta}=\frac{a b x_{(i)}^{3}}{3}\left[1-\left\{1-\left[1-e^{-\left(\alpha x_{(i)}+\frac{\theta}{2} x_{(i)}^{2}+\frac{\beta}{3} x_{(i)}^{3}\right)}\right]^{a}\right\}^{b}-\frac{i}{n+1}\right]
\end{aligned}
$$

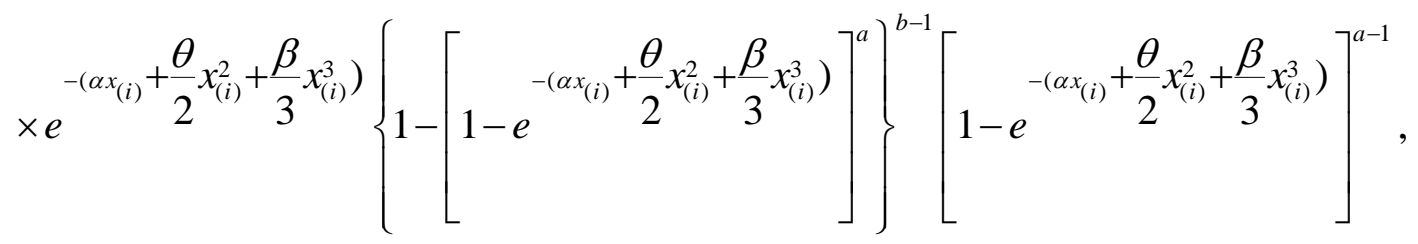

$$
\begin{aligned}
& \frac{\partial Q}{\partial a}=b\left[1-\left\{1-\left[1-e^{-\left(\alpha x_{(i)}+\frac{\theta}{2} x_{(i)}^{2}+\frac{\beta}{3} x_{(i)}^{3}\right)}\right]^{a}\right\}^{b}-\frac{i}{n+1}\right] \ln \left[1-e^{-\left(\alpha x_{(i)}+\frac{\theta}{2} x_{(i)}^{2}+\frac{\beta}{3} x_{(i)}^{3}\right)}\right] \\
& \times\left\{1-\left[1-e^{-\left(\alpha x_{(i)}+\frac{\theta}{2} x_{(i)}^{2}+\frac{\beta}{3} x_{(i)}^{3}\right)}\right]^{a}\right\}^{b-1}\left[1-e^{-\left(\alpha x_{(i)}+\frac{\theta}{2} x_{(i)}^{2}+\frac{\beta}{3} x_{(i)}^{3}\right)}\right]^{a}
\end{aligned}
$$

and

$$
\begin{aligned}
& \frac{\partial Q}{\partial b}=\left[1-\left\{1-\left[1-e^{-\left(\alpha x_{(i)}+\frac{\theta}{2} x_{(i)}^{2}+\frac{\beta}{3} x_{(i)}^{3}\right)}\right]^{a}\right\}^{b}-\frac{i}{n+1}\right]\left\{1-\left[1-e^{-\left(\alpha x_{(i)}+\frac{\theta}{2} x_{(i)}^{2}+\frac{\beta}{3} x_{(i)}^{3}\right)}\right]^{a}\right\}^{b} \\
& \times \ln \left\{1-\left[1-e^{-\left(\alpha x_{(i)}+\frac{\theta}{2} x_{(i)}^{2}+\frac{\beta}{3} x_{(i)}^{3}\right)}\right]^{a}\right\}
\end{aligned}
$$

The estimates of the parameters are obtained by equating the above equations to zero. Although the proposed estimators cannot be expressed in closed form, they can be obtained through the use of an appropriate numerical solution algorithm.

Method 2 (Weighted Least Squares Estimators). The weighted least squares estimators can be obtained by minimizing

$$
\sum_{i=1}^{n} w_{i}\left(G\left(Y_{(i)}\right)-\frac{i}{n+1}\right)^{2},
$$

with respect to the unknown parameters, where

$$
w_{i}=\frac{1}{V\left(G\left(Y_{(i)}\right)\right)}=\frac{(n+1)^{2}(n+2)}{i(n-i+1)} \text {. }
$$


Therefore, in case of $K Q H R$ distribution the weighted least squares estimators of $\alpha, \theta, \beta, a$ and $b$, say $\alpha_{W L S E}, \theta_{W L S E}, \beta_{W L S E}, a_{W L S E}$ and $\hat{b}_{W L S E}$ respectively, can be obtained by minimizing

$$
\sum_{i=1}^{n} w_{i}\left[1-\left\{1-\left[1-e^{-\left(\alpha x+\frac{\theta}{2} x^{2}+\frac{\beta}{3} x^{3}\right)}\right]^{a}\right\}-\frac{i}{n+1}\right]^{b}
$$

with respect to the unknown parameters only.

\section{Maximum Likelihood Estimators}

In this section we consider the maximum likelihood estimators (MLE's) of $K Q H R$ distribution. Let $\Phi=(\alpha, \theta, \beta, a, b)^{T}$, in order to estimate the parameters $\alpha, \theta, \beta, a$ and $b$ of kumaraswamy quadratic hazard rate distribution, let $x_{1}, \ldots, x_{n}$ be a random sample of size $n$ from $K Q H R$ $(x ; \alpha, \theta, \beta, a, b)$ then the log likelihood function can be written as

$$
\begin{aligned}
L= & n \ln a+n \ln b+\sum_{i=1}^{n} \ln \left(\alpha+\theta x_{(i)}+\beta x_{(i)}^{2}\right)-\alpha \sum_{i=1}^{n} x_{(i)}-\frac{\theta}{2} \sum_{i=1}^{n} x_{(i)}^{2}-\frac{\beta}{3} \sum_{i=1}^{n} x_{(i)}^{3} \\
& +(a-1) \sum_{i=1}^{n} \ln \left[1-e^{-\left(\alpha x+\frac{\theta}{2} x^{2}+\frac{\beta}{3} x^{3}\right)}\right]+(b-1) \sum_{i=1}^{n} \ln \left\{1-\left[1-e^{-\left(\alpha x+\frac{\theta}{2} x^{2}+\frac{\beta}{3} x^{3}\right)}\right]^{a}\right\}
\end{aligned}
$$

Differentiating $L$ with respect to each parameter $\alpha, \theta, \beta, a$ and $b$ and setting the result equals to zero, we obtain maximum likelihood estimates. The partial derivatives of $L$ with respect to each parameter or the score function is given by

where

$$
U_{n}(\Phi)=\left(\frac{\partial L}{\partial \alpha}, \frac{\partial L}{\partial \theta}, \frac{\partial L}{\partial \beta}, \frac{\partial L}{\partial a}, \frac{\partial L}{\partial b}\right)
$$

$$
\begin{aligned}
& \frac{\partial L}{\partial \alpha}= \sum_{i=1}^{n} \frac{1}{\left(\alpha+\theta x_{(i)}+\beta x_{(i)}^{2}\right)}-\sum_{i=1}^{n} x_{(i)}+(a-1) \sum_{i=1}^{n} \frac{x_{(i)} e^{-\left(\alpha x_{(i)}+\frac{\theta}{2} x_{(i)}^{2}+\frac{\beta}{3} x_{(i)}^{3}\right)}}{\left[1-e^{-\left(\alpha x+\frac{\theta}{2} x^{2}+\frac{\beta}{3} x^{3}\right)}\right]} \\
&+a(b-1) \sum_{i=1}^{n} \frac{x_{(i)} e^{-\left(\alpha x+\frac{\theta}{2} x^{2}+\frac{\beta}{3} x^{3}\right)[}\left[1-e^{-\left(\alpha x+\frac{\theta}{2} x^{2}+\frac{\beta}{3} x^{3}\right)}\right]^{a-1}}{\left\{1-\left[1-e^{-\left(\alpha x+\frac{\theta}{2} x^{2}+\frac{\beta}{3} x^{3}\right)}\right]^{a}\right\}}=0
\end{aligned}
$$




$$
\begin{aligned}
& \frac{\partial \log L}{\partial \theta}=\sum_{i=1}^{n} \frac{x_{(i)}}{\left(\alpha+\theta x_{(i)}+\beta x_{(i)}^{2}\right)}-\frac{1}{2} \sum_{i=1}^{n} x_{(i)}^{2}+(a-1) \sum_{i=1}^{n} \frac{x_{(i)}^{2} e^{-\left(\alpha x_{(i)}+\frac{\theta}{2} x_{(i)}^{2}+\frac{\beta}{3} x_{(i)}^{3}\right)}}{2\left[1-e^{-\left(\alpha x+\frac{\theta}{2} x^{2}+\frac{\beta}{3} x^{3}\right)}\right]} \\
& +(b-1) \sum_{i=1}^{n} \frac{x_{(i)}^{2} e^{-\left(\alpha x+\frac{\theta}{2} x^{2}+\frac{\beta}{3} x^{3}\right)\left[1-e^{-\left(\alpha x+\frac{\theta}{2} x^{2}+\frac{\beta}{3} x^{3}\right)}\right]^{a-1}}}{2\left\{1-\left[1-e^{-\left(\alpha x+\frac{\theta}{2} x^{2}+\frac{\beta}{3} x^{3}\right)}\right]^{a}\right\}}=0,
\end{aligned}
$$

and

$$
\begin{aligned}
& \frac{\partial \log L}{\partial \beta}=\sum_{i=1}^{n} \frac{x_{(i)}^{2}}{\left(\alpha+\theta x_{(i)}+\beta x_{(i)}^{2}\right)}-\frac{1}{3} \sum_{i=1}^{n} x_{(i)}^{3}+(a-1) \sum_{i=1}^{n} \frac{x_{(i)}^{3} e^{-\left(\alpha x_{(i)}+\frac{\theta}{2} x_{(i)}^{2}+\frac{\beta}{3} x_{(i)}^{3}\right)}}{3\left[1-e^{-\left(\alpha x+\frac{\theta}{2} x^{2}+\frac{\beta}{3} x^{3}\right)}\right]} \\
& +(b-1) \sum_{i=1}^{n} \frac{x_{(i)}^{3} e^{-\left(\alpha x+\frac{\theta}{2} x^{2}+\frac{\beta}{3} x^{3}\right)}\left[1-e^{-\left(\alpha x+\frac{\theta}{2} x^{2}+\frac{\beta}{3} x^{3}\right)}\right]^{a-1}}{3\left\{1-\left[1-e^{-\left(\alpha x+\frac{\theta}{2} x^{2}+\frac{\beta}{3} x^{3}\right)}\right]^{a}\right\}}=0, \\
& \frac{\partial \log L}{\partial a}=\frac{n}{a}+\sum_{i=1}^{n} \ln \left[1-e^{-\left(\alpha x+\frac{\theta}{2} x^{2}+\frac{\beta}{3} x^{3}\right)}\right]
\end{aligned}
$$$$
+\sum_{i=1}^{n} \frac{\ln \left[1-e^{-\left(\alpha x+\frac{\theta}{2} x^{2}+\frac{\beta}{3} x^{3}\right)}\right]\left[1-e^{-\left(\alpha x+\frac{\theta}{2} x^{2}+\frac{\beta}{3} x^{3}\right)}\right]^{a}}{\left\{1-\left[1-e^{-\left(\alpha x+\frac{\theta}{2} x^{2}+\frac{\beta}{3} x^{3}\right)}\right]^{a}\right\}}=0,
$$ 


$$
\frac{\partial \log L}{\partial b}=\frac{n}{b}+\sum_{i=1}^{n} \ln \left\{1-\left[1-e^{-\left(\alpha x+\frac{\theta}{2} x^{2}+\frac{\beta}{3} x^{3}\right)}\right]^{a}\right\}=0
$$

By solving this nonlinear system of equations (6.2) - (6.6), these solutions will yield the ML estimators for $\alpha, \theta, \beta, a$ and $\hat{b}$. For the five parameters kumaraswamy quadratic hazard rate distribution $K Q H R(\alpha, \theta, \beta, a, b, x)$ pdf all the second order derivatives exist. Thus we have the inverse dispersion matrix is given by

$$
\begin{aligned}
& \left(\begin{array}{c}
\alpha \\
\theta \\
\beta \\
a \\
\hat{b}
\end{array}\right) \sim N\left[\left(\begin{array}{c}
\alpha \\
\theta \\
\beta \\
a \\
b
\end{array}\right),\left(\begin{array}{ccccc}
V_{\alpha \alpha} & V_{\alpha \theta} & V_{\alpha \beta} & V_{\alpha a} & V_{\alpha b} \\
V_{\theta \alpha} & V_{\theta \theta} & V_{\theta \beta} & V_{\theta a} & V_{\theta b} \\
V_{\beta \alpha} & V_{\beta \theta} & V_{\beta \beta} & V_{\beta a} & V_{\beta b} \\
V_{a \alpha} & V_{a \theta} & V_{a \beta} & V_{a a} & V_{a b} \\
V_{b \alpha} & V_{b \theta} & V_{b \beta} & V_{b a} & V_{b b}
\end{array}\right)\right] . \\
& V^{-1}=-E\left[\begin{array}{ccccc}
V_{\alpha \alpha} & V_{\alpha \theta} & V_{\alpha \beta} & V_{\alpha a} & V_{\alpha b} \\
& & & & \\
V_{b \alpha} & V_{b \theta} & V_{b \beta} & V_{b a} & V_{b b}
\end{array}\right]
\end{aligned}
$$

where

$$
\begin{aligned}
& V_{\alpha \alpha}=\frac{\partial^{2} L}{\partial \alpha^{2}}, V_{\theta \theta}=\frac{\partial^{2} L}{\partial \theta^{2}}, V_{\beta \beta}=\frac{\partial^{2} L}{\partial \beta^{2}}, V_{a a}=\frac{\partial^{2} L}{\partial a^{2}}, V_{b b}=\frac{\partial^{2} L}{\partial b^{2}} \\
& V_{\alpha \theta}=\frac{\partial^{2} L}{\partial \alpha \partial \theta}, V_{a \alpha}=\frac{\partial^{2} L}{\partial \alpha \partial a}, V_{\beta b}=\frac{\partial^{2} L}{\partial \beta \partial b}, V_{a b}=\frac{\partial^{2} L}{\partial a \partial b}, V_{\beta \alpha}=\frac{\partial^{2} L}{\partial \beta \partial \alpha}
\end{aligned}
$$

By solving this inverse dispersion matrix these solutions will yield asymptotic variance and covariances of these ML estimators for $\alpha, \theta, \beta, a$ and $\hat{b}$. . Using (6.8), we approximate $100(1-\gamma) \%$ confidence intervals for $\alpha, \theta, \beta, a$ and $b$ are determined respectively as

$$
\alpha \pm z_{\frac{\gamma}{2}} \sqrt{V_{\alpha \alpha}}, \theta \pm z_{\frac{\gamma}{2}} \sqrt{V_{\theta \theta}}, \beta \pm z_{\frac{\gamma}{2}} \sqrt{V_{\beta \beta}} \text { and } \lambda \pm z_{\frac{\gamma}{2}} \sqrt{V_{\lambda \lambda}}
$$

where $z_{\gamma}$ is the upper $100 \gamma$ the percentile of the standard normal distribution.

\subsection{Application}


SPSS buit-in dataset "Breast Cancer Survival" is used to obtain fit The quadratic hazard rate distribution $(Q H R)$ distribution. The ML estimates of the parameters of The quadratic hazard rate distribution $(Q H R)$ distribution are given in the table 2. We have also given the AIC and negative of log likelihood function to decide about the suitability of the model.

\begin{tabular}{cccccccc}
\hline & \multicolumn{5}{c}{ Parameters (SE in Parenthesis) } & \multirow{2}{*}{$-2 \log (\mathrm{LF})$} & AIC \\
\cline { 2 - 6 } Variable & $\mathbf{a}$ & $\mathbf{b}$ & $\boldsymbol{\alpha}$ & $\boldsymbol{\beta}$ & $\boldsymbol{\theta}$ & & \\
\hline $\begin{array}{c}\text { Survival } \\
\text { Time in } \\
\text { years }\end{array}$ & 10347.77 & 8488.49 & 3.34 & 115.87 & 47.75 & & \\
\hline
\end{tabular}

\section{References}

1. Amoroso, L. (1925). Ricerche intorno alla curva dei redditi. Ann. Math., 2, 123-159.

2. Bain. L.J (1974). Analysis for the linear failure-rate life-testing distribution, Technometrics, 16(4), 551-559.

3. Cordeiro G. M. and de Castro, M. (2011). A new family of generalized distributions. Journal of Statistical Computation and Simulation, 81, 883-898.

4. Cordeiro, G. M., Ortega, E.M.M. and Nadarajah, S. (2010). The Kumaraswamy Weibull distribution with application to failure data. Journal of the Franklin Institute, 347, 1399-1429.

5. Cordeiro, G.M., Nadarajah, S. and Ortega, E. M. M. (2012). The Kumaraswamy Gumbel distribution. Statistical Methods and Applications, 21(2), 139-168.

6. Elbatal. I. (2013). Kumaraswamy Generalized linear failure rate. Indian Journal of Computational and Applied Mathematics, 1(1), 61-78.

7. Elbatal. I. (2013). The Kumaraswamy Exponentiated Pareto Distribution. Economic Quality Control, 28(1), 1-8.

8. Eugene, N., Lee, C. and Famoye, F. (2002). Beta-normal distribution and its applications. Communications in Statistics: Theory and Methods, 31, 497-512.

9. Good, I. J. (1953). The population frequencies of the species and the estimation of population parameters. Biometrika, 40, 237-260.

10. Gupta, R. C., Gupta, P.L. and Gupta. R.D. (1998). Modeling failure time data by Lehman alternatives. Commun. Stat. Theory Methods, 27, 887-904.

11. Hoskings, J. R. M., and J. R. Wallis (1987). Parameter and quantile estimation for the generalized Pareto distribution. Technometrics, 29, 339-349.

12. Jones, M. C. (2009). A beta- type distribution with some tractability advantages. Statistical Methodology, 6, 70-81.

13. Johnson, N. L., Kotz, S and N. Balakrishnan (1995). Continuous Univariate Distribution, V(2), 2nd edition, New York, Wiley. 
14. Kumaraswamy, P. (1980). Generalized probability density function for double bounded random-processes. Journal of Hydrology, 462, 79-88.

15. McDonald, J. B. (1984). Some generalized functions for the size distribution of income. Econometrica, 52, 647-663.

16. Nadarajah, S., G. M. Cordeiro, and E. M. M. Ortega. (2011). General results for the Kumaraswamy-G distribution. J. Stat. Comput. Simulation. doi:10.1080/00949655.2011.562504.

17. Pascoa, A.R.M., Ortega, E.M.M. and Cordeiro, G.M. (2011). The Kumaraswamy generalized gamma distribution with application in survival analysis. Statistical Methodology, 8, 411-433.

18. SPSS Inc. Released 2007. SPSS for Windows, Version 16.0. Chicago, SPSS Inc.

19. Saulo, H., Lea $\sim$ o, J. and Bourguignon, M. (2012). The Kumaraswamy BirnbaumSaunders Distribution. Journal of Statistical Theory and Practice, 6, 745-759.

20. Swain. J,S. Venkatraman. S, and Wilson. J (1988). Least squares estimation of distribution function in Johnson's translation system. Journal of Statistical Computation and Simulation, 29, 271-297. 
Appendix-I: Plots of density function (pdf) of Kumaraswamy quadratic hazard rate distribution for various values of $a, b, \alpha, \beta$ and $\theta$

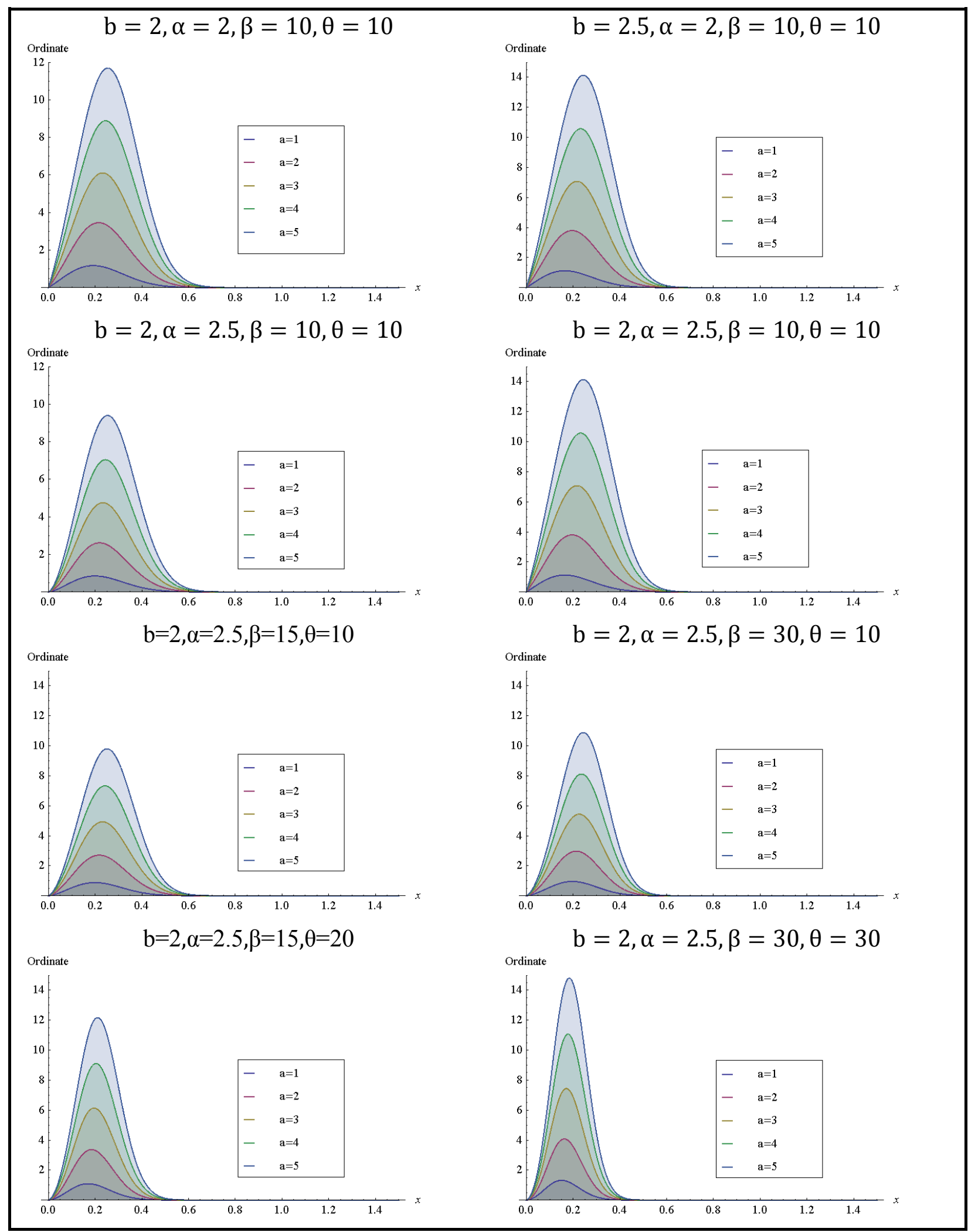


Appendix-II: Plots of hazard rate function of Kumaraswamy quadratic hazard rate distribution for various values of $a, b, \alpha, \beta$ and $\theta$

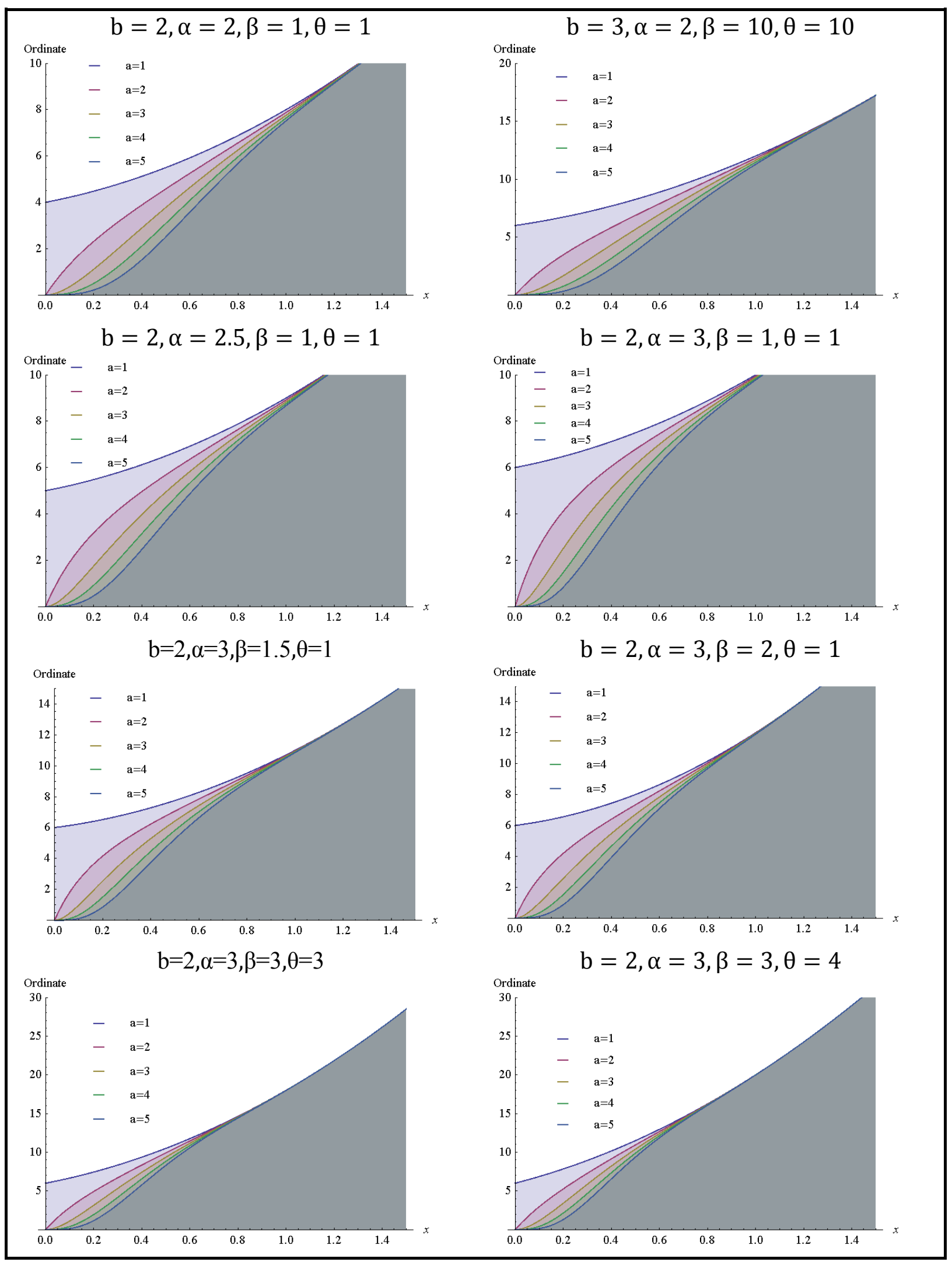

